\title{
Compliance program como herramienta en la lucha contra la corrupción en Ecuador
}

\author{
Compliance program as a tool to fight \\ against corruption in Ecuador
}

\author{
José Ignacio Miranda Cifuentes \\ Investigador Jurídico, Gladys Terán Abogados Asociados
}

Artículo Original (Revisión)

RFJ, No. 4, 2018, pp. 37-52, ISSN 2588-0837

RESUMEN: los programas de cumplimiento normativo o compliance program tienen como objetivo evitar el cometimiento de delitos dentro de una organización determinada, por este motivo, frente a la problemática de la corrupción en nuestro país, estos programas surgen como una opción justificada para identificar y sancionar las prácticas corruptas dentro de una organización. Sin embargo, en nuestro país no se encuentra regulada la institución del compliance program, por lo que resulta menester implementar esta institución en nuestro ordenamiento jurídico para, desde una política de prevención eficaz, beneficiarnos de la práctica del compliance y sus efectos.

PALABRAS CLAVE: cumplimiento normativo, compliance program, corrupción, Estado, empresa.

ABSTRACT: the programs of compliance or compliance program aim to avoid the commission of crimes within a given organization, for this reason, in the face of the problem of corruption in our country, these programs emerge as a justified option to identify and sanction corrupt practices within an organization. However, the institution of the compliance program is not regulated in our country, which is why it is necessary to implement this institution in our legal system so that, from an effective prevention policy, we can benefit from the practice of compliance and its effects.

KEY WORDS: regulatory compliance, compliance program, corruption, State, company. 


\section{INTRODUCCIÓN}

La finalidad de este artículo es introducir a la discusión doctrinaria una institución de gran importancia que, desde una cultura de prevención, evita el cometimiento de delitos dentro de una organización determinada, sea en el sector público o privado, como es el Compliance Program, por lo que, ante los últimos casos de corrupción en nuestro país, en los que se ven inmersos funcionarios públicos de alto nivel, surgen ciertas dudas respecto a la postura del Estado frente a esta problemática, ya que, al existir, normas, controles y entidades públicas que luchan contra la corrupción, la apreciación obvia de la ciudadanía es que estos mecanismos de control no son suficientes. Por este motivo, en el presente trabajo se analizará la institución del Compliance Program como una herramienta eficaz para evitar el cometimiento de delitos, entre ellos, delitos de corrupción.

En este contexto, en una primera parte de la presente investigación se estudiará la institución del compliance de manera general, sobre sus antecedentes y el contenido en sí de estos programas. En segundo lugar, se analizará la incidencia del compliance program en dos de los ámbitos jurídicos donde se presenta con mayor fuerza, según los efectos positivos que surgen en materia penal y en contratación pública, a través de un estudio comparado, tomando como fuente la legislación española (respecto a la responsabilidad penal de las personas jurídicas) así como las directrices de la Unión Europea y el sistema federal de contratación pública en EEUU. Por último, se propondrá un modelo de compliance aplicable al Ecuador encaminado a luchar, desde la prevención, contra la corrupción.

\section{CONCEPTO Y NATURALEZA JURIDICA DEL COMPLIANCE PROGRAM}

Para empezar con el presente trabajo es necesario entender al compliance como un "conjunto de políticas y estrategias previstas por cada empresa para prevenir, detectar y evitar prácticas de corrupción dentro de su estructura" Corporate Compliance Survey (2005, p. 1759), es decir, se trata de un mecanismo de prevención de cometimiento de delitos dentro de una organización determinada.

Estas estrategias se ven plasmadas en códigos de conducta, reglamentos internos, códigos de buen gobierno corporativo, etc. 
Para prevenir el cometimiento de delitos el compliance program presenta dos fines generales: (i) sancionar la falta de conductas leales, transparentes y éticas; y (ii) determinar responsabilidad dentro de la organización, es decir identificar al personal que delinquió. En este sentido, dentro de la doctrina se distinguen dos sistemas de compliance aplicados según las necesidades propias de cada organización: centralizado y descentralizado. Wellner, P (2005)

El sistema centralizado se caracteriza por la existencia de un tercero, que no forma parte de la organización, a cargo del desarrollo y control del programa, en este sistema se designa un oficial de cumplimiento o compliance officer. Este oficial es el encargado de desarrollar todo el programa anticorrupción, de la investigación y documentación de estos incidentes, así como la supervisión y seguimiento a las necesidades regulatorias de cada empresa. Fine, M. (2013) Además, el sistema descentralizado se encuentra dirigido por directivos de la empresa o senior managers que se encargan directamente de desarrollar e implementar el programa basado en "líneas de negocio que [...]deben responder al presidente de la compañía, y posiblemente a un comité de auditoría, [...] a su vez, debe establecer los objetivos y proporcionar guías". Fine, M. (2013, p.12) Como se evidencia en el primer caso, existe una organización ajena a la empresa encargada de crear los programas de cumplimiento y establecer los mecanismos de control y sanción, así como, auditorias periódicas a base de los riesgos y necesidades de la empresa que contrata sus servicios para implementar el compliance ${ }^{1}$; además, en el caso del sistema descentralizado, son sus directivos los encargados de realizar estas funciones.

Por lo antes dicho, se colige que cada modelo aplicable responde a la estructura y organización propia de la empresa según los riesgos que se derivan de las actividades que esta presta, en tal virtud, el compliance officer debe ser un profesional que al no formar parte de la organización, es un tercero encargado del desarrollo del programa, su contenido según de la investigación de riesgos de la empresa, así como el control interno de cumplimiento del programa; asimismo, en el sistema descentralizado, estas atribuciones son encargadas a los altos directivos de la empresa.

1 Por lo general, el compliance officer es un estudio jurídico especializado en la creación, implementación y control de los programas de cumplimiento. 
Al contener el compliance program, un estudio de riesgos que se derivan de la actividad propia de la organización, sea esta pública o privada, no se puede decir con precisión cuál es el contenido exacto de un programa de cumplimiento, lo que puede ser visto, según Gordon, S., como una ventaja en la medida que su carácter abierto permitiría su continua adaptación a la realidad cambiante de la actividad empresarial (2010). Sin embargo, Gómez-Jara Díez, C. ha establecido como requisitos mínimos respecto al contenido del compliance program los siguientes:

(1) existencia de un código de conducta escrito; (2) supervisión de los esfuerzos de cumplimiento por parte del personal altamente cualificado; (3) no delegación de poderes discrecionales de las autoridades administrativas en personal con posible tendencia delictiva; (4) comunicación efectiva de los estándares y procedimientos contenidos en los códigos de conducta; (5) reforzamiento mediante procedimientos disciplinarios; (6) adopción de medidas adecuadas tras la detección de la infracción. (2006, p. 77)

Así, por ejemplo, una constructora que contrata con el Estado no presenta los mismos riesgos que una importadora de insumos tecnológicos, según los delitos que su personal pueda cometer. Así mismo, estos programas son aplicables a instituciones del Estado para evitar delitos de corrupción contra la administración ${ }^{2}$ o la implementación del compliance dentro de organizaciones, movimientos o partidos políticos. Serrano Espelta, H (2015)

Independientemente del programa que más se ajuste a las necesidades de la organización, cada programa debe ser "claro , conciso y completo" Shapiro,K. ${ }^{3}$ (2003 p. 40), por el contrario, estos programas serían ineficaces, por lo que resulta menester que los programas sean

2 Ver: COIP Art. 278 Peculado; Art.279 Enriquecimiento ilícito; Art 280 Cohecho; Art. 281 Concusión; etc.

3 Sostiene que para que los programas de compliance sean "claros, concisos y completos" deben contener:

(i) Un análisis de riesgos acorde con la actividad económica de la empresa. (ii) Los programas deben ser promulgados dentro de la empresa para que todos sus integrantes la conozcan. (iii) Un sistema de sanciones y canalización de denuncias dentro de la organización, y; (iv) La designación de un órgano autónomo superior encargado de la supervisión y control.

Ver: Shapiro,KarenL."10Stepstoabetterday:Thekeycomponentsof compliance". Business Law Today. Volume13,No.1.AmericanBarAssociation,(2003). Traducción libre. 
promulgados dentro de la empresa para que todo el personal lo conozca y lo acate, caso contrario, los sistemas de cumplimiento son solo un conjunto de buenas intenciones, ya que estos deben ir acompañados de procedimientos internos y normas de control que aseguren su efectivo cumplimiento. Nieto, M. (2013)

En tal sentido, al surgir esta práctica de varios riesgos potenciales, sus ámbitos de aplicación son extensos según la necesidad regulatoria de la empresa, entre ellos, Nieto Martin A. (2013) destaca: la prevención de riesgos laborales, la protección de datos, prevención de lavado de activos, prevención contra actos de corrupción, el abuso de mercado o el mercado de valores, etc.

En este sentido, podemos concluir que al existir un programa efectivo de compliance dentro de una empresa existen también varias ventajas e incentivos que se presentan en la organización: en primer lugar se crea un mejor ambiente laboral ya que se consolida un sentimiento de pertenencia a la empresa por sus buenas prácticas organizacionales, por lo que mejora la producción de la compañía en tanto sus trabajadores conocen el código de conducta, los procedimientos a seguir y la autoridad a quien acudir en caso de detectar una conducta ilícita; la imagen de la compañía con los clientes mejora, ya que estos buscan confianza y seguridad; de la misma manera, gracias a las prácticas de conductas honestas apegadas a la ley, el país en general percibe más ganancias por el incremento de inversionistas extranjeros, y, a su vez, mayor confianza de la población en general. Desio, P. (2014)

Esta herramienta no es tan novedosa, ya que la regulación de compliance surge en Estados Unidos a raíz de la promulgación de la Foreign Corrupt Practices Act (FCPA) o Ley de Prácticas Corruptas en el Extranjero, el 20 de diciembre de 1977. Esta ley, como lo menciona McGreal, E. (2009), presentaba dos objetivos principales: (i) sancionar a personas (naturales o jurídicas) que provean cualquier pago, promesa o recompensa a un oficial del gobierno extranjero por obtener o retener un negocio; (ii) sancionar a organizaciones que realicen pagos a un tercero, sea este intermediario, agente o contratista, sabiendo que para obtener cierto beneficio le pagaría de igual manera a un oficial del gobierno extranjero.

En ese contexto, el compliance program o "cumplimiento normativo" ha tomado mayor importancia en las dos últimas décadas debido a los casos de corrupción que se mantienen presentes en la región, esta pro- 
blemática surge a raíz del desarrollo económico en los países generado por la globalización que promueve el incremento de intercambios comerciales, así como, el incremento en mayor volumen de información sobre las actividades comerciales y políticas a nivel nacional e internacional. López Pagán, J. y Villoria M. (2009)

Frente a esta problemática ${ }^{4}$ y gracias al desarrollo normativo por parte de EEUU, en la región, de a poco se ha ido incrementando estas prácticas de prevención, en este sentido, Latinoamérica ha desarrollado mecanismos y ha implementado leyes para combatir la corrupción y tratar de erradicarla. Por lo que, a manera de antecedente, encontramos la Convención Interamericana Contra la Corrupción, en Caracas, Venezuela, ${ }^{5}$ que en su artículo 2 establece como propósitos de dicha convención los siguientes:

1. Promover y fortalecer el desarrollo, por cada uno de los Estados Partes, de los mecanismos necesarios para prevenir, detectar, sancionar y erradicar la corrupción; y

2. Promover, facilitar y regular la cooperación entre los Estados Partes a fin de asegurar la eficacia de las medidas y acciones para prevenir, detectar, sancionar y erradicar los actos de corrupción en el ejercicio de las funciones públicas y los actos de corrupción específicamente vinculados con tal ejercicio. (OEA, 1996). En nuestro país, encontramos como deber primordial del Estado, garantizar a sus habitantes una sociedad libre de corrupción'. Como podemos observar, de la normativa citada anteriormente, en la actualidad existen mandatos legales nacionales como internacionales, que sustenten la creación y desarrollo de los programas de cumplimiento normativo o compliance, por lo que resulta ineficaz

$4 \quad$ Ninguno de los veintiséis países que conforman Latinoamérica alcanza un lugar dentro de los diez primeros puestos con menor índice de corrupción en el mundo. El país mejor puntuado de la región es Uruguay, que ocupa el vigésimo primer puesto, con una calificación de 74 puntos. Mientras que el país latinoamericano con la calificación más baja es Venezuela, que obtuvo 8 puntos, y el puesto número 158. Transparencia Internacional. Índice de Percepción de la Corrupción 2017. http://goo.gl/cNqvga.

5 Esta se promulgó mediante la Convención de la Organización de Estados Americanos (OEA), firmada por 22 de los 35 miembros de la Organización en marzo de 1996.

6 Art. 3 de la Constitución. - Son deberes primordiales del Estado: 8. Garantizar a sus habitantes el derecho a una cultura de paz, a la seguridad integral y a vivir en una sociedad democrática y libre de corrupción. 
que frente a la problemática de la corrupción en la región las organizaciones, públicas o privadas, no cuenten con estas herramientas, cuyos efectos y beneficios serán tratados a continuación.

\section{EFECTOS JURÍDICOS DEL "COMPLIANCE PROGRAM" FRENTE A LA RESPONSABILIDAD PENAL DE LAS PERSO- NAS JURIDICAS SEGÚN EL CASO ESPAÑOL}

Para nuestro estudio, encontramos en el sistema procesal penal español que cuando una empresa se ve inmersa en un proceso penal, al acreditar la existencia de un programa de cumplimiento eficaz la responsabilidad penal se elimina o se atenúa según el caso, estas ventajas varían dependiendo de las personas que comenten el delito, el momento del cometimiento del ilícito o según el estadio procesal en que se acredite la existencia del programa.

De esta manera, para que la responsabilidad penal de la persona jurídica se elimine, según el artículo 31 del Código Penal español, es necesario que el órgano de administración haya adoptado y ejecutado con eficacia, antes de la comisión del delito, modelos de organización y gestión que incluyen las medidas de vigilancia y control idóneas para prevenir delitos de la misma naturaleza o para reducir de forma significativa el riesgo de su comisión. ${ }^{7}$

$7 \quad$ Art 31: A) Delitos cometidos por representantes legales, o por aquellos que actuando individualmente o como integrantes de un órgano de la persona jurídica, están autorizados para tomar decisiones en nombre de la persona jurídica u ostentan facultades de organización y control dentro de la misma. (Art. 31 bis 2 CP). En estos supuestos, la persona jurídica quedará exenta de responsabilidad sí se cumplen las siguientes condiciones:

1. ${ }^{a}$ El órgano de administración ha adoptado y ejecutado con eficacia, antes de la comisión del delito, modelos de organización y gestión que incluyen las medidas de vigilancia y control idóneas para prevenir delitos de la misma naturaleza o para reducir de forma significativa el riesgo de su comisión.

2. ${ }^{\text {a }}$ La supervisión del funcionamiento y del cumplimiento del modelo de prevención implantado ha sido confiada a un órgano de la persona jurídica con poderes autónomos de iniciativa y de control o que tenga encomendada legalmente la función de supervisar la eficacia de los controles internos de la persona jurídica.

(El Legislador se refiere al oficial o comité de cumplimiento o compliance officer) 3. ${ }^{\mathrm{a}}$ Los autores individuales han cometido el delito eludiendo fraudulentamente los modelos de organización y de prevención. 
En este sentido, el efecto de la acreditación es que se extingue la facultad que la empresa responda penalmente y esta responsabilidad recaiga sobre la persona natural miembro de la organización que realizó la conducta penalmente reprochable. De igual manera, una vez implementado el programa es necesario que este sea confiado a un órgano de la persona jurídica con poderes autónomos de iniciativa y de control o que tenga encomendada legalmente la función de supervisar la eficacia de los controles internos de la persona jurídica. (compliance officer).

Además, frente a la responsabilidad penal de las personas jurídicas, para que la pena sea atenuada, es necesario prestar todas las facilidades de cooperación dentro del proceso penal, así como establecer, antes del comienzo del juicio oral, medidas eficaces para prevenir y descubrir los delitos que en el futuro pudieran cometerse con los medios o bajo la cobertura de la persona jurídica. ${ }^{8}$

Como podemos evidenciar, en ambos supuestos el legislador español se está refiriendo a la implementación en las empresas de planes

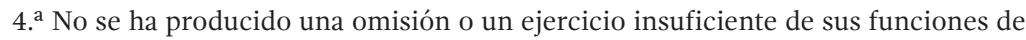
supervisión, vigilancia y control por parte del órgano de cumplimiento...

B) Actuaciones cometidas por quienes están sometidos a la autoridad de las personas físicas mencionadas en el apartado A) (Art. 31 bis 4 CP)

La persona jurídica quedará exenta de responsabilidad sí, antes de la comisión del delito, ha adoptado y ejecutado eficazmente un modelo de organización y gestión que resulte adecuado para prevenir delitos de la naturaleza del que fue cometido o para reducir de forma significativa el riesgo de su comisión.

8 Art 31: son circunstancias atenuantes de la responsabilidad penal de las personas jurídicas las siguientes:

1. La acreditación parcial de las circunstancias eximentes. (Art. 31 bis 2 y 4 CP)

2. Haber realizado, con posterioridad a la comisión del delito y a través de sus representantes legales, las siguientes actividades (art. 31 quater CP):

a) Haber procedido, antes de conocer que el procedimiento judicial se dirige contra ella, a confesar la infracción a las autoridades.

b) Haber colaborado en la investigación del hecho aportando pruebas, en cualquier momento del proceso, que fueran nuevas y decisivas para esclarecer las responsabilidades penales dimanantes de los hechos.

c) Haber procedido en cualquier momento del procedimiento y con anterioridad al juicio oral a reparar o disminuir el daño causado por el delito.

d) Haber establecido, antes del comienzo del juicio oral, medidas eficaces para prevenir y descubrir los delitos que en el futuro pudieran cometerse con los medios o bajo la cobertura de la persona jurídica. 
de prevención de riesgos o compliance, la existencia de estos programas eximen la responsabilidad penal de la persona jurídica si la empresa ha implementado un programa de compliance efectivo antes del cometimiento del ilícito, mientras que sí se implementa el plan después del conocimiento del cometimiento del delito con fines de evitar que estas conductas se vuelva a repetir, la pena se atenúa.

En este sentido, Osvaldo Artaza menciona que el fundamento de esta ventaja en materia penal obedece a que la responsabilidad penal propia de la empresa se basa en un defecto de organización, por tal razón, la responsabilidad de la empresa no deriva delito cometido por uno de sus ejecutivos o representantes, sino que es consecuencia del incumplimiento o cumplimiento imperfecto de los deberes de dirección y supervisión de la entidad, que se traducen fundamentalmente en su incapacidad para prevenir su utilización para la comisión de delitos. (2013)

A base de lo antes mencionado, la sentencia STS 154/2016, de la Corte Superior Española, respecto a la responsabilidad penal de las personas jurídicas, menciona que el fin del compliance se basa en "la exigencia del establecimiento y correcta aplicación de medidas de control eficaces que prevengan e intenten evitar, en lo posible, la comisión de infracciones delictivas por quienes integran la organización” (Tribunal Superior de Justicia, 2016)

En este contexto, constatada la realización del hecho delictivo, lo que ha de demostrarse en el proceso penal por parte de fiscalía es que la persona jurídica, de manera continuada, no había adoptado medidas eficaces para prevenir hechos delictivos de la especie del que se ha cometido y en la forma en que concretamente ha aparecido. Asimismo, es la persona jurídica la que debe demostrar en el proceso, que implementó un programa eficaz que evite el cometimiento del delito por el que se imputa, que su análisis de riesgos ha sido correcto y no le resultaba exigible desarrollar medidas de prevención en relación con riesgos penales no relevantes según los riesgos propios de la empresa al desarrollar su actividad económica. Artaza, O. (2013)

A base de lo dicho anteriormente, podemos decir que los programas de cumplimiento normativo constituyen el conjunto de medidas que la empresa debe implementar para contar con una organización proba y no ser responsable penalmente o ver rebajada su pena en el caso en el que alguno de sus integrantes (administradores, directivos, trabajadores, etc.) cometa un delito en el desempeño de sus funciones. 


\section{COMPLIANCE PROGRAM Y SUS EFECTOS EN CONTRA- TACIÓN PÚBLICA}

Podemos afirmar que la eficacia de estos modelos no queda reducida al ámbito penal, sino que son reflejo de una cultura de cumplimiento y ética empresarial que abarca todas las actividades de la persona jurídica, en este sentido encontramos que, en materia de contratación pública, la exigencia de estos programas en procesos de licitación ha surgido cada vez con más fuerza frente a la problemática de la corrupción.

Para iniciar tomaremos como ejemplo el caso europeo, que exige la implementación de estos planes para contratar con el Estado. ${ }^{9}$ Las directivas de 2014 exigen que los licitadores demuestren la implementación de programas eficaces de compliance con el objetivo de prevenir y luchar contra la corrupción, las actividades delictivas y las distorsiones de la competencia. También, encontramos que en Estados Unidos, que es el país pionero en temas de cumplimiento normativo y el que ha alcanzado un mayor desarrollo en la materia, desde el año 2008 ha implementado un sistema de contratación en virtud del cual "para poder ser siquiera una entidad capaz de contratar con la administración pública federal, debe contar con un programa de cumplimiento anti-corrupción". (López Donaire, M. 2016, p.73)

Como lo menciona Hartmann, W. (2012), estos programas referidos a la contratación federal se fundamentan en cuatro objetivos básicos: integridad al tratar con funcionarios federales (prohibición de promesas, dádivas o recompensas; prohibición de ofrecer puestos en el sector privado tras abandonar el servicio público, etc.); honestidad en el intercambio de información (prohibición de cualquier tipo de falsedad documental, etc.); consolidación de relaciones éticas con terceras partes (prohibición de kick-back ${ }^{10}$, etc.); fomentar la competencia leal.

9 El 26 de febrero de 2014 el Parlamento Europeo y el Consejo aprobaron tres nuevas Directivas 2014/23/UE, relativa a la adjudicación de contratos de concesión; 2014/24/UE, sobre contratación pública y 2014/25/UE, relativa a la contratación que operan en los sectores del agua, la energía, los transportes y los servicios postales.

10 Con la expresión "Kick-back" se alude básicamente a aquellos supuestos en los que el administrador o el representante de una empresa, en el momento de cerrar un contrato, acuerda con la otra parte contratante que esta le entregue una comisión que, posteriormente, repercute al alza en el importe total que acaba pagando su empresa al adquirir el producto o servicio contratados. Kaikati, G (200,p. 54) 
Para cumplir con estos objetivos, cualquier empresa que contrate con el gobierno federal debe disponer de un sistema de compliance que contenga los siguientes elementos:

Un mapa de riesgo, un código ético y formación adecuada sobre el mismo; revisión periódica para asegurar la ética de cumplimiento; un mecanismo para reportar las conductas ilegales; determinadas instrucciones que incentiven a los empleados a reportar; auditorías internas o externas; acciones disciplinarias frente a la conducta ilegal; reportar a tiempo a la administración pública; colaboración plena con las agencias gubernamentales que se encarguen de perseguir las conductas ilegales. (López Donaire, M. 2016, p.27)

Como podemos evidenciar de los casos expuestos anteriormente, tanto en Europa como en EEUU, la exigencia del compliance en materia de contratación se ha presentado con más fuerza. Nuestra región no debe ser la excepción frente al desarrollo de políticas de prevención de delitos encaminados a la lucha contra la corrupción ya que los grandes casos de corrupción presentados en la región han surgido, justamente, en materia de contratación pública, por lo que los Estados deberían promover estas prácticas y replicarlas, no obstante, también sería deseable, como señala NIETO MARTÍN (2014), que las Administraciones Públicas adoptaran en su seno medidas de prevención eficaces, inspirado en los programas de compliance de las empresas privadas, lo que él denomina public compliance ${ }^{11}$ para detectar o mitigar los riesgos no solo desde el ámbito penal.

\section{APLICACIÓN DEL COMPLIANCE PROGRAM EN EL ECUA- DOR EN LA LUCHA CONTRA LA CORRUPCIÓN}

La corrupción es uno de los mayores problemas que azota a nuestra sociedad, cada vez son más sofisticadas las formas de delinquir, así como los actores que se involucran en estos casos. Ya que como lo menciona Fons, J. Frente a esta dinámica los delitos se van perfeccionando, lo que genera varios efectos nocivos para el país. En primer

11 Establece que dentro de la administración pública se deben replicar los programas de compliance con su contenido y sus objetivos, ya que detrás de un acto de corrupción, generalmente, hay un funcionario público involucrado. Ver: NIETO MARTÍN, A: "Public Compliance. Prevención de la corrupción en administraciones públicas y partidos políticos”. Ed. Tirant lo Blanc, 2014. 
lugar, el Estado se ve afectado en la recaudación de impuestos, considerando que no existe declaración alguna por parte de los grupos que operan de manera ilícita. (2003)

En segundo lugar, para poder encontrar a los responsables de los delitos cometidos, se debe activar el aparataje estatal y sus colaboradores; es decir, el gasto público destinado a seguridad crece en correspondencia al alza de índices de inseguridad y delincuencia. Por último, la participación de funcionarios públicos y de figuras políticas en ciertos casos de corrupción, crea desconfianza respecto de estas instituciones de manera general y, por ende, de todo el sistema. Lo que a su vez crea inseguridad jurídica y, en consecuencia, falta de interés por parte de compañías e inversionistas extranjeros, de ahí la necesidad de regular los programas de compliance en el país. Bedoya M. (2017)

Por lo antes dicho, y frente a esta problemática, una de las medidas que se deben implementar en el país es la exigencia del compliance en materia de contratación pública como uno de los requisitos para contratar con el Estado, en este sentido, el programa de cumplimiento normativo, siguiendo los lineamientos internacionales, deberá evitar al menos el cometimiento de delitos contra la administración pública. Para lograr este propósito se debe establecer un mecanismo de recepción de denuncias dentro de la empresa; así como los mecanismos para detectar, a base de auditorías y controles internos, cualquier evento sospechoso que pueda ser catalogado como la comisión de un delito.

Sin embargo, esta implementación no resultaría del todo atractiva, ya que ni a las empresas ni a las entidades públicas les interesaría denunciar actos delictivos de corrupción, que surgen en el seno de sus actividades y que podrían involucrar a altos directivos o funcionarios públicos, si no es posible la aplicación de alguna circunstancia de favorabilidad que beneficie la situación penal de la persona jurídica. En ese sentido encontramos en el Código Orgánico Integral Penal (COIP) un régimen de responsabilidad penal aplicable a las Personas Jurídicas, es decir, cuando se incurre en una conducta de las tipificadas en el COIP, el proceso penal puede dirigirse no solamente contra el autor persona natural, sino también contra la persona jurídica. Sin embargo, podríamos afirmar que este tratamiento normativo en el COIP es incompleto, ya que no existen actualmente en nuestro sistema penal circunstancias modificatorias de la pena que favorezcan la situación penal, en ciertos casos, de las personas jurídicas. 
Por esta razón debería incorporarse la aplicación del compliance en materia penal, para completar la teoría delictiva respecto a la responsabilidad penal de las personas jurídicas en Ecuador, ya que como se mencionó, lo que se sanciona no es el cometimiento en sí del delito, sino un defecto de organización dentro de la empresa, en este sentido, las personas jurídicas, deberían poder contar con mecanismos que atenúen o eximen su responsabilidad penal con el objetivo que la responsabilidad recaiga contra la persona natural que cometió el ilícito, permitiendo a la empresa demostrar, a través de la existencia de un programa de compliance, que no existe tal falla o defecto en la organización de la empresa, ya que al demostrar solvencia organizacional, quedaría fuera del poder sancionador del Estado. Araujo, P. (2014)

\section{CONCLUSIONES}

Frente a la problemática de la corrupción en la región los programas de cumplimiento son creados y puestos en práctica para prevenir y detectar la mala conducta, así como para asegurar que las actividades empresariales se realicen de conformidad con las leyes aplicables. Dicho modelo se convierte de forma unívoca en el instrumento válido para que la organización pueda acreditar el ejercicio de las tareas de supervisión y control encomendadas, y a su vez, recibir los beneficios de la aplicación del compliance program. En este sentido, en el Ecuador es necesaria una reforma al COIP, que integre la posibilidad de acreditar la existencia de un programa de cumplimiento normativo como una circunstancia de favorabilidad penal que atenué la pena o elimine la responsabilidad penal de las personas jurídicas.

Asimismo, el Estado debe fomentar e impulsar la autorregulación en las empresas privadas, ya que la adopción de programas de compliance tiene un mayor alcance en el ámbito de la contratación pública reduciendo el riesgo de cometer delitos, por lo que resulta necesario en el Ecuador una reforma que establezca como requisito indispensable para contratar con el Estado, que la empresa cuente con un programa de compliance efectivo para evitar actos de corrupción desde una cultura de prevención.

Como se abordó en el presente trabajo, los beneficios del compliance son varios, por lo que la práctica del compliance resulta sumamente productiva, no solo en aspectos económicos para la empresa, sino también para la sociedad en general, ya que estos mecanismos de prevención 
podrán posicionar a las empresas en rankings más altos, y garantizar transparencia en los servicios brindados. En tal virtud, gracias a una correcta política de prevención que integre las necesidades de los principales actores económicos (empresa, Estado y sociedad) se puede acabar, o la menos reducir los índices de corrupción en nuestro país.

\section{REFERENCIAS BIBLIOGRÁFICAS}

Araujo, P. (2014). La nueva teoría del delito económico y empresarial en el Ecuador. Quito: Corporación de Estudios y Publicaciones.

Artaza, O. (2013). Sistemas de prevención de delitos o programas de cumplimiento. Breve descripción de las reglas técnicas de gestión del riesgo empresarial y su utilidad en sede jurídico penal" polit crim. Vol. 8, No 16 (diciembre 2013), [http://www.politicacriminal.cl/ Vol_08/n_16/Vol8N16A6.pdf]

Bedoya M. (2017). Compliance: su evolución y desafíos en Ecuador ¿̇hacia dónde ir?. Universidad San Francisco de Quito. USFQ Law Review

\section{Código Penal Español}

Convención Interamericana Contra la Corrupción (1996).

Corporate Compliance, Committee ABA Section of Business Law. Corporate Compliance Survey. The Business Lawyer. Vol. 60, No. 4 (2005). Traducción libre.

Desio, P. (2014). An Overview of the Organizational Guidelines. United States Sentencing Commission.Traducción libre.

Fine, E. (2003). Putting the pieces together: A game plan for an effective Compliance structure. Business Law Today. Volume 13, No. 1. American Bar Association. Traducción libre.

Fons, J. (2003). The case for compliance: Now it's a necessity, not an option. American Business Law. Volume 13, No. 1. American Bar Association. Traducción libre. 
Gómez-Jara Díez, C. (2006). La responsabilidad penal de las Empresas en los EEUU, Sevilla: Ed. Fundación Ramón Areces.

Gordon, S. (2010). Implementation of Effective Compliance and Ethics Programs and the Federal Sentencing Guidelines en: VV. AA., Corporate Compliance Answer Book (2010). Estados Unidos: Ed. Practising Law Institute. Capítulo 2 disponible en Web: http://www.pli.edu/ product_files/booksamples/26962_sample2.pdf.

Hartmann, W. (2012). Effective Government Contract Compliance Strategies in a Constantly Evolving Legal Landscape, en: Government Contracts Compliance.

Kaikati, G. et al. (2000). The Price of International Business Morality: Twenty Years Under the Foreign Corrupt Practices Act. Journal of Business Ethics. Volumen 26, No. 3. Springer, p. 213. Traducción libre.

López Donaire, M. Los Programas De Compliance y Su Reflejo En La Contratación Pública GABILEX No 6 junio 2016. www.gabilex.jccm.es

López Pagán, J. y Villoria, M. (2009). Globalización, corrupción y convenios internacionales dilemas y propuestas para España. Madrid: Real Instituto Elcano.

McGreal, E. (2009). Corporate Compliance Survey. The Business Lawyer. Volumen 65, No. 1. American Bar Association. Traducción libre.

Nieto Martin, A. (2013). Problemas Fundamentales Del Cumplimiento Normativo En El Derecho Penal. «Good citizens corporations: Programas de cumplimiento normativo en entidades públicas». Ref. POII100105-614.

Nieto Martin, A. (2014). Public Compliance. Prevención de la corrupción en administraciones públicas y partidos políticos. Ed. Tirant lo Blanc.

Serrano Espelta, H. et al. (2015). Práctica de Compliance en Latinoamérica. Estado actual de la legislación anticorrupción y otras. Grupo Interamericano de la práctica de compliance. Primera ed. Bogotá: Brigard \& Urrutia Abogados. 
Shapiro, K. (2003). 10 Steps to a better day: The key components of compliance. Business Law Today. Volume 13, No. 1. American Bar Association. Traducción Libre.

STS 154/2016 España Tribunal Superior Penal

Wellner, P. (2005). Effective compliance programs and corporate criminal prosecutions, N27, Cardozo Law Review, New York.

Recibido: 17 de junio de 2018

Aceptado: 8 de noviembre de 2018

José Ignacio Miranda Cifuentes: Investigador Jurídico, Gladys Terán Abogados Asociados.

Correo electrónico: jimc92.jm@gmail.com 